\section{Predictors of positive endoscopic findings in hospitalized patients with occult gastrointestinal bleeding}

\author{
Neil Sengupta, Elliot B. Tapper, \\ Vilas R. Patwardhan, Anthony J. Lembo, \\ Joseph D. Feuerstein \\ Division of Gastroenterology, Beth Israel \\ Deaconess Medical Center, Boston, MA, \\ USA
}

\section{Abstract}

Anemia with occult gastrointestinal bleeding (OGIB) is a common reason for inpatient gastroenterology consultation and endoscopy. However, the utility of inpatient endoscopy in this setting is unclear. The aim of this paper is to determine variables that predict positive endoscopic examinations for inpatients presenting with anemia and OGIB. We performed a prospective observational study of consecutive hospitalized patients between March 2013 and April 2014 with anemia and OGIB. For patients undergoing inpatient endoscopic evaluation, logistic regression was used to determine which variables were associated with detecting etiology of OGIB. An occult bleeding score (OBS) was created using receiver operating characteristics and area under the curve (AUC) analysis to predict detection of bleeding source and need for endoscopic intervention. Of the 74 patients identified, 55 (74\%) underwent endoscopic evaluation, of whom 28 patients had a source of OGIB identified. Patients with malignancy (OR $7.25,95 \% \mathrm{CI}$ 1.06-144) were more likely to have a bleeding source identified on endoscopy, whereas patients with higher admission hemoglobin levels were less likely to have a detected source (OR 0.62, 95\%CI 0.41-0.86). The OBS was constructed by assigning a point each for malignancy and admission hemoglobin $<8.2 \mathrm{~g} / \mathrm{dL}$. The OBS predicted detection of etiology of bleeding and endoscopic intervention with an AUC of 0.79 and 0.75 . The yield of endoscopy in determining a bleeding source in patients with OGIB is significant particularly in patients with malignancy or a low hemoglobin level. A simple score can assist in determining whether inpatient endoscopy is warranted in this population.

\section{Introduction}

Occult gastrointestinal bleeding (GIB) refers to the clinical presentation of a positive stool fecal occult blood test (FOBT) with or without iron deficiency anemia in the absence of overt bleeding. ${ }^{1}$ Although only recommended in the context of colon cancer screening, ${ }^{2}$ FOBT is frequently ordered in the inpatient setting due to anemia, ${ }^{3}$ and is a common reason for inpatient gastroenterology consultation. ${ }^{4}$ Because of its false positive results, inpatient guaiac testing commonly leads to negative endoscopic testing, ${ }^{4,5}$ and has been shown to have little beneficial impact on inpatient clinical management. ${ }^{6}$ In fact, despite endoscopic evaluation with upper esophagogastroduodenoscopy (EGD) and colonoscopy, the source of bleeding can remain undetected in patients with occult GIB in up to $52 \%$ of patients. ${ }^{7}$

In such cases where initial endoscopic evaluation is unrevealing, current guidelines recommend consideration of further studies such as capsule endoscopy and enteroscopy. $2,8,9$ However, although occult GIB is a common clinical scenario in the hospital, there is limited data regarding which risk factors predict either detecting the bleeding source on inpatient endoscopy, or the need for endoscopic intervention. In addition, recent data have described risk factors for increased 30-day hospital readmissions and rebleeding for patients with overt GIB. ${ }^{10}$ There is limited data on whether patients with occult bleeding have increased hospital readmission and rebleeding rates, and whether endoscopic intervention or localization during index hospitalization can reduce these rates.

Our primary aims were to determine the yield of endoscopy in patients with anemia and occult GIB and whether clinical or laboratory parameters on admission could predict patients who were high or low risk for having either a source of occult GIB detected on endoscopic evaluation, or require endoscopic intervention. Other aims were to report all-cause 30-day hospital readmissions and rebleeding in a population of hospitalized patients with anemia and occult GIB.

\section{Materials and Methods}

As described elsewhere, ${ }^{10,11}$ we performed a prospective observational cohort study of consecutive patients admitted with or developing GIB in the hospital. Data were collected on a total of 750 patients admitted to Beth Israel Deaconess Medical Center (BIDMC, Boston, MA, USA) from March 2013 to April 2014. Patients were included in this study if the gastroenterology service was consulted for anemia and occult GIB, as defined by a positive stool guaiac test in the setting of anemia. Patients with overt GIB ( $\mathrm{n}=676)$ as defined by presence of hematemesis, coffee ground eme-
Correspondence: Joseph D. Feuerstein, Division of Gastroenterology, Beth Israel Deaconess Medical Center, 110 Francis Street 8E, Boston, MA 02215, USA

Tel.: +1.617.632.8623 - Fax: +1.617.632.9199.

E-mail: Jfeuerst@bidmc.harvard.edu

Key words: Gastrointestinal hemorrhage; occult blood; guaiac; gastrointestinal endoscopy.

Contributions: NS, JDF, acquisition of data, study concept and design, study supervision; NS, EBT, drafting of the manuscript, statistical analysis; NS, JDF, EBT, VRP, analysis and interpretation of data; NS, JDF, AJL, EBT, VRP, critical revision of the manuscript for important intellectual content.

Conflict of interest: the authors declare no potential conflict of interest.

Received for publication: 3 July 2015. Accepted for publication: 1 September 2015.

This work is licensed under a Creative Commons Attribution NonCommercial 3.0 License (CC BYNC 3.0).

(c) Copyright N. Sengupta et al., 2015

Licensee PAGEPress, Italy

Gastroenterology Insights 2015; 6:6097

doi:10.4081/gi.2015.6097

sis, melena, or hematochezia were excluded from this study.

We obtained baseline demographic information and the following clinical data via medical record review during hospitalization: comorbidities, home medications, and initial laboratory studies. Baseline hemoglobin values were recorded by review of prior clinic notes. We recorded the details of in-hospital management, transfusion requirements, endoscopic procedures and interventions, and etiology of bleeding. Inpatients were classified as having iron deficiency, if the transferrin saturation was less than $20 \%$, or if the ferritin levels were less than $40 \mathrm{ng} / \mathrm{mL}$. For all etiologies, the bleeding source was defined based on the presence of stigmata of hemorrhage on endoscopic evaluation. Endoscopic interventions included the use of endoclips, electrocautery, epinephrine, or argon plasma coagulation. The timing of initial procedure and choice of endoscopic therapy was left to the discretion of the attending gastroenterologist, as there was no structured protocol for evaluation and management of these patients.

Discharge data included hospital length of stay (LOS), and discharge medications. Patients were mailed a letter to inform them of the study and the plan to contact them by telephone to complete a survey. An investigator (NS, JDF, VRP) contacted all patients via telephone at 30 days after discharge to determine 
all interval emergency department (ED) visits and hospital readmissions. Rebleeding events as well as the reason for interval readmissions were confirmed via review of our online medical record. Deaths occurring in follow up were confirmed by reviewing the medical record and online death registries. ${ }^{12}$

The study was approved by the BIDMC Institutional Review Board.

\section{Statistical analysis}

Categorical variables are reported as percentages, and continuous variables are reported as medians (interquartile ranges). Comparisons between groups for categorical data were made with Fisher's exact test. Continuous data were compared using the 2sample t test for normally distributed variables and Wilcoxon rank-sum test for non-normally distributed variables. Univariable and multivariable logistic regression were used to determine associations of variables to localizing a source of occult GIB. An occult bleeding score (OBS) was constructed using admission hemoglobin and presence of active malignancy. The optimal hemoglobin cutoff was determined by the receiver operating characteristic curve (ROC). The ability of the OBS to predict localization of bleeding and endoscopic intervention was assessed by area under the curve (AUC) analysis. Univariable logistic regression was used to report associations between clinical variables and both 30-day readmissions and rebleeding. All analyses were performed using JMP Pro 10.0 (SAS Institute, Cary, NC, USA).

\section{Results}

Of the 750 patients who the GI service was consulted on for GIB during the study period, seventy-four inpatients were identified who were admitted with or developed anemia and occult GIB. Seventy-four percent $(n=55)$ underwent an inpatient endoscopic procedure and 19 (26\%) were managed conservatively without endoscopic evaluation. None of the patients who were managed conservatively developed overt GIB during hospitalization. Patients who underwent an endoscopic procedure had a higher difference between their previous baseline hemoglobin prior to hospitalization, and their nadir hemoglobin during hospitalization ( $3.2 \mathrm{~g} / \mathrm{dL}$ vs $2.2 \mathrm{~g} / \mathrm{dL}, \mathrm{P}=0.03)$. Patients on systemic anticoagulation were not more likely to have an inpatient endoscopic evaluation for occult GIB and anemia (40\%, $\mathrm{n}=22 / 55$ vs $32 \%, \mathrm{n}=6 / 19 ; \mathrm{P}=0.59$ ). Sixty-two percent $(n=46 / 74)$ of the cohort had no identified source of bleeding during the hospitalization. One patient died during hospitalization due to septic shock who did not undergo endoscopic evaluation. This patient did not develop overt GIB. The median age of the cohort was 74

Table 1. Baseline demographics.

\begin{tabular}{|c|c|c|c|c|}
\hline Clinical variables & Cohort $(n=74)$ & Procedure performed $(n=55)$ & No procedure $(n=19)$ & P value \\
\hline Age, median (IQR) & $74(62-84)$ & $74(61-84)$ & $71(62-86)$ & 0.84 \\
\hline Male sex, $\mathrm{n}(\%)$ & $41(55)$ & $31(56)$ & $10(53)$ & 0.80 \\
\hline Tobacco, n (\%) & $17(23)$ & $5(26)$ & $12(22)$ & 0.75 \\
\hline In-hospital GIB, n (\%) & $12(16)$ & $8(42)$ & $4(7)$ & 0.001 \\
\hline Number of comorbidities, median (IQR) & $10(8-13)$ & $10(8-13)$ & $12(7-16)$ & 0.38 \\
\hline $\begin{array}{l}\text { Comorbidities, } \mathrm{n}(\%) \\
\text { Hypertension } \\
\text { Coronary artery disease } \\
\text { Atrial fibrillation } \\
\text { Congestive heart failure } \\
\text { Cerebrovascular disease } \\
\text { Renal failure } \\
\text { Active malignancy } \\
\text { Cirrhosis } \\
\text { History of GIB }\end{array}$ & $\begin{array}{l}58(78) \\
30(41) \\
19(26) \\
20(27) \\
16(22) \\
32(43) \\
10(14) \\
10(14) \\
30(41)\end{array}$ & $\begin{array}{c}43(78) \\
23(42) \\
14(25) \\
13(24) \\
14(25) \\
25(45) \\
6(11) \\
8(15) \\
24(44)\end{array}$ & $\begin{array}{l}15(79) \\
7(37) \\
5(26) \\
7(37) \\
2(11) \\
7(37) \\
4(21) \\
2(11) \\
6(32)\end{array}$ & $\begin{array}{c}1.0 \\
0.79 \\
1.0 \\
0.37 \\
0.21 \\
0.60 \\
0.27 \\
1.0 \\
0.42\end{array}$ \\
\hline Iron deficiency, n (\%) & $40(54)$ & $33(60)$ & $7(37)$ & 0.11 \\
\hline $\begin{array}{l}\text { Home medications, n (\%) } \\
\text { Anticoagulants } \\
\text { Aspirin }\end{array}$ & $\begin{array}{l}28(38) \\
41(55)\end{array}$ & $\begin{array}{l}22(40) \\
28(51)\end{array}$ & $\begin{array}{c}6(32) \\
13(68)\end{array}$ & $\begin{array}{l}0.59 \\
0.28\end{array}$ \\
\hline $\begin{array}{l}\text { Etiology, n (\%) } \\
\text { Arteriovenous malformation } \\
\text { Dieulafoy lesion } \\
\text { Diverticular } \\
\text { Variceal } \\
\text { Gastritis } \\
\text { GAVE } \\
\text { Portal gastropathy } \\
\text { Duodenal ulcer } \\
\text { Gastric ulcer } \\
\text { No source }\end{array}$ & $\begin{array}{l}7(9) \\
3(4) \\
1(1) \\
1(1) \\
2(3) \\
3(4) \\
3(4) \\
2(3) \\
6(8) \\
46(62)\end{array}$ & $\begin{array}{l}7(13) \\
3(5) \\
1(2) \\
1(2) \\
2(4) \\
3(5) \\
3(5) \\
2(4) \\
6(11) \\
27(49)\end{array}$ & $\begin{array}{c}\text { N/A } \\
- \\
- \\
- \\
- \\
- \\
- \\
- \\
- \\
19(100)\end{array}$ & $\begin{array}{l}\text { N/A } \\
- \\
- \\
- \\
- \\
- \\
- \\
- \\
- \\
-\end{array}$ \\
\hline $\begin{array}{l}\text { Management, } \mathrm{n}(\%) \\
\text { Endoscopic intervention } \\
\text { Blood transfusion } \\
\text { Fresh frozen plasma } \\
\text { Intensive unit care } \\
\text { Length of stay, median IQR } \\
\text { Nadir hemoglobin, g/dL } \\
\text { Difference between Baseline and Nadir Hgb, g/dL }\end{array}$ & $\begin{array}{c}11(15) \\
54(73) \\
7(9) \\
19(26) \\
5(3-9) \\
7.0(6.4-7.6) \\
2.8(1.7-4.2)\end{array}$ & $\begin{array}{c}11(20) \\
41(75) \\
7(13) \\
14(26) \\
5(3-9) \\
6.9(6.4-7.6) \\
3.2(1.8-4.5)\end{array}$ & $\begin{array}{c}0(0) \\
13(68) \\
0(0) \\
5(26) \\
5(3-10) \\
7.1(6.6-7.8) \\
2.2(1.6-2.9)\end{array}$ & $\begin{array}{c}\mathrm{N} / \mathrm{A} \\
0.77 \\
0.18 \\
1.0 \\
0.55 \\
0.62 \\
0.03\end{array}$ \\
\hline
\end{tabular}

$\mathrm{IQR}$, interquartile range; GIB, gastrointestinal bleeding; GAVE, gastric antral vascular ectasias; Hgb, hemoglobin. 
years (IQR 62-84). Fifty-four percent $(n=40 / 74)$ of patients had evidence of iron deficiency at the time of hospitalization. Demographics and occult GIB etiology are summarized in Table 1.

\section{Diagnostic procedures and their yields}

The source of occult bleeding was detected in $36 \%(\mathrm{n}=18 / 50)$ of patients undergoing inpatient EGD $(n=50)$, whereas the source of bleeding was detected in only $12 \%(n=3 / 25)$ of patients undergoing colonoscopy after a non-diagnostic EGD. Median time to EGD was 48 hours (range 6-168 hours), and the median time to colonoscopy was 72 hours (range 24 to 168 hours). A source of bleeding was detected in an additional 3 patients undergoing capsule endoscopy ( $n=8)$ after non-diagnostic EGD and colonoscopy. Two of these three patients subsequently went on to having an enteroscopy, one of whom had a bleeding source confirmed on enteroscopy. Two additional patients underwent an enteroscopy without having a preceding capsule endoscopy, neither of whom had a positive source detected.

\section{Localization of bleeding}

For the 55 patients who had an endoscopic evaluation, logistic regression was performed to determine variables associated with detecting a bleeding source (Table 2). Patients with active malignancy at time of occult bleeding (OR 7.25, 95\%CI 1.06-144, P=0.04), and patients requiring inpatient blood transfusion (OR 4.25, 95\% CI 1.13-20.9, $\mathrm{P}=0.03$ ) were more likely to have a bleeding source detected. Patients with higher admission hemoglobin levels (OR 0.62 per-unit, 95\% CI 0.41-0.86, $\mathrm{P}=0.003)$ and nadir hemoglobin levels during hospitalization (OR 0.65 per-unit, 95\%CI 0.41-
$0.92, \mathrm{P}=0.01$ ) were less likely to have an identified bleeding source. Age and use of anticoagulation were not associated with detection of a bleeding source.

On multivariable logistic regression adjusting for presence of active malignancy and blood transfusion, higher admission hemoglobin values were associated with a decreased risk of detecting a bleeding source (OR 0.65, 95\%CI 0.40-0.96, $\mathrm{P}=0.04$ ).

\section{Occult bleeding score}

An occult bleeding score (OBS) was constructed for patients undergo3ing endoscopy by assigning a point each for active malignancy and admission hemoglobin levels less than $8.2 \mathrm{~g} / \mathrm{dL}$. The association between the OBS and localization of bleeding and endoscopic intervention is depicted in Figure 1. For patients with an OBS of $0,6 \%(n=1 / 18)$ had a source of occult bleeding detected. No patients with an OBS of 0 required endoscopic intervention. The OBS predicted detection of bleeding source with an AUC of 0.79 ( $95 \%$ CI $0.67-0.87$ ), and endoscopic intervention with an AUC of 0.75 (95\%CI 0.64-0.82).

\section{Post discharge outcomes}

Twenty-five patients (34\%) were either readmitted to a hospital or required an ED visit within 30 days of initial hospital discharge (21 patients with hospital readmissions and 4 with only ED visits). The most common reason for readmission or ED visit was recurrent gastrointestinal bleeding $(n=9)$. Other common reasons for hospitalization or ED visits included flare of congestive heart failure $(n=4)$, infection ( $\mathrm{n}=3)$, and decompensated liver disease $(n=2)$. Of the 9 patients with recurrent bleeding within 30 days, 4 patients presented

Table 2. Variables associated with localizing a source of occult bleeding for patients undergoing an inpatient endoscopic procedure $(n=55)$.

\begin{tabular}{lccc} 
Variables & \multicolumn{3}{c}{ Localived source of bleeding } \\
& OR & $95 \%$ CI & P value \\
Age & 1.04 & $0.99-1.08$ & 0.09 \\
Female sex & 1.38 & $0.47-4.10$ & 0.55 \\
\hline Systemic anticoagulation & 1.36 & $0.46-4.06$ & 0.58 \\
Intensive unit care & 1.22 & $0.36-4.21$ & 0.75 \\
\hline Coronary artery disease & 2.17 & $0.73-6.62$ & 0.16 \\
Iron deficiency & 1.36 & $0.46-4.13$ & 0.58 \\
\hline Active malignancy & 7.25 & $1.06-144$ & 0.04 \\
Cirrhosis & 2.25 & $0.49-12.0$ & 0.30 \\
\hline Blood transfusion & 4.25 & $1.13-20.9$ & 0.03 \\
Admit hemoglobin & 0.62 & $0.41-0.86$ & 0.003 \\
\hline Nadir hemoglobin & 0.65 & $0.41-0.92$ & 0.01 \\
\hline
\end{tabular}

OR, odds ratio, $\mathrm{Cl}$; confidence interval.

Table 3. Post-hospital discharge outcomes for entire cohort on univariable logistic regression (n=74).

\begin{tabular}{|c|c|c|c|c|c|c|}
\hline \multirow[t]{2}{*}{ Variables } & \multicolumn{3}{|c|}{ 30-Day ED or readmission ( $\mathrm{n}=25)$} & \multicolumn{3}{|c|}{ 30-Day Rebleeding ( $\mathrm{n=9}$ ) } \\
\hline & OR & $95 \% \mathrm{CI}$ & P value & OR & $95 \% \mathrm{CI}$ & P value \\
\hline Age & 0.99 & $0.95-1.03$ & 0.57 & 0.99 & $0.95-1.05$ & 0.86 \\
\hline Female sex & 2.58 & $0.97-7.13$ & 0.06 & 2.81 & $0.68-14.3$ & 0.17 \\
\hline Systemic anticoagulation & 0.89 & $0.32-2.38$ & 0.82 & 1.37 & $0.31-5.65$ & 0.66 \\
\hline Number of comorbidities & 1.12 & $1.01-1.25$ & 0.04 & 1.17 & $1.02-1.37$ & 0.03 \\
\hline Coronary artery disease & 2.62 & $0.98-7.22$ & 0.05 & 3.42 & $0.83-17.4$ & 0.09 \\
\hline Renal failure & 1.34 & $0.50-3.56$ & 0.56 & 3.00 & $0.72-15.2$ & 0.14 \\
\hline Active malignancy & 2.20 & $0.56-8.77$ & 0.25 & 2.03 & $0.27-10.4$ & 0.44 \\
\hline Aspirin use & 5.33 & $1.82-18.2$ & 0.002 & 3.19 & $0.71-22.5$ & 0.14 \\
\hline Admission hemoglobin & 0.93 & $0.72-1.17$ & 0.53 & 0.81 & $0.54-1.14$ & 0.24 \\
\hline Iron deficiency & 1.13 & $0.43-3.01$ & 0.81 & 0.64 & $0.15-2.65$ & 0.54 \\
\hline Nadir Hemoglobin & 0.90 & $0.67-1.18$ & 0.46 & 0.74 & $0.44-1.14$ & 0.19 \\
\hline Procedure performed & 0.83 & $0.28-2.57$ & 0.75 & 3.06 & $0.51-58.9$ & 0.25 \\
\hline Diagnosis confirmed & 1.34 & $0.50-3.73$ & 0.53 & 1.56 & $0.36-6.49$ & 0.54 \\
\hline
\end{tabular}

ED, Emergency Department; OR, odds ratio; CI, confidence interval. 
with new onset overt bleeding after initially presenting with occult GIB. Two of these patients presented with overt UGIB [one patient with hematemesis due to gastric antral vascular ectasias (GAVE) and one patient with melena due to a duodenal ulcer]. The patient with rebleeding due to a duodenal ulcer did not have an upper EGD during the index hospitalization, whereas the patient with GAVE was identified during EGD on index hospitalization. The other two patients had overt LGIB with subsequent negative colonoscopies. Of the 5 patients with recurrent occult GIB, 2 patients had a capsule endoscopy during the hospital readmission showing angioectasias in the jejunum with stigmata of hemorrhage. One patient without endoscopic evaluation during initial hospitalization was found to have severe gastritis on hospital readmission. One patient with known history of GAVE presented with recurrent anemia requiring repeat argon plasma coagulation. Finally, one patient had persistent obscure, occult GIB. On univariable logistic regression, an increased number of comorbidities (OR 1.12, 95\%CI 1.01-1.25; $\mathrm{P}=0.04$ ) were associated with increased risk of 30-day hospital readmissions. Other variables associated with 30-day readmissions are detailed in Table 3. Clinical variables associated with recurrent GIB are detailed in Table 3. Increased number of comorbidities were associated with increased risk of rebleeding ( $\mathrm{OR}$ 1.17, 95\%CI: 1.02-1.37, $\mathrm{P}=0.03$ ). Having an inpatient endoscopic evaluation was not associated with a reduced risk of recurrent bleeding (OR 3.06, 95\% CI 0.51-58.9, $\mathrm{P}=0.25$ ). Use of systemic anticoagulation was not associated with an increased risk of recurrent bleeding (OR 1.37, 95\%CI 0.31-5.65, $\mathrm{P}=0.66$ ). Five patients (7\%) died prior to the 30 day study call. Four patients died from metastatic cancer, and one patient died from septic shock.

A Proportion of Patients With a Localized
Bleeding Source By Occult Bleeding Score

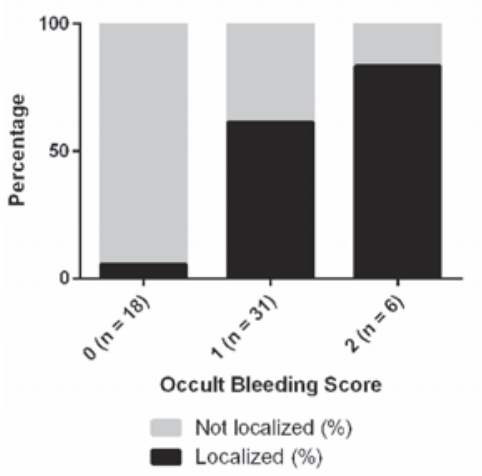

\section{Discussion}

Fecal occult blood testing in the setting of anemia is commonly performed in the inpatient setting, and frequently leads to gastroenterology consultation and inpatient diagnostic evaluation. ${ }^{6,13}$ Although there is data suggesting that advanced age and mean corpuscular volume may predict a positive endoscopic evaluation in patients with iron deficiency anemia, ${ }^{14,15}$ there is limited data on which hospitalized patients with anemia and a positive fecal occult blood test (FOBT) have either positive findings on endoscopic evaluation or need endoscopic intervention. In this prospective study, we demonstrate that hospitalized patients with concern for occult GIB who have active malignancy and lower admission hemoglobin levels are more likely to have a positive source of GIB detected on endoscopic evaluation. More importantly, a simple scoring tool was able to identify low risk patients with anemia and occult GIB who were unlikely to benefit from an endoscopic evaluation or need endoscopic intervention.

In a previous study of patients with iron deficiency anemia, patients with positive FOBT were more likely to have endoscopic lesions identified than those without. ${ }^{15}$ Rockey and colleagues studied patients with positive FOBT without iron deficiency. ${ }^{7}$ In that population, upper gastrointestinal lesions were identified more frequently than colonic lesions with $29 \%$ and $22 \%$ of cases being localized by EGD and colonoscopy respectively. We extend and add to the prior literature with a simple scoring system that could predict whether occult bleeding will be localized on endoscopy. In addition, as no patient with a score of 0 required an endoscopic intervention, our results suggest that stable inpatients with

\section{B}

Proportion of Patients With An Endoscopic Intervention By Occult Bleeding Score

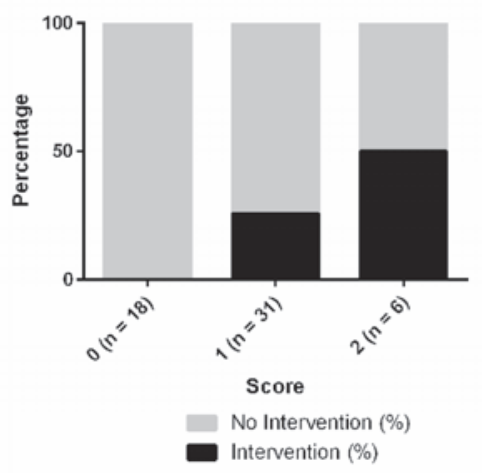

occult GIB without transfusion requirements or active cancer may have their workup deferred to the outpatient setting, thereby decreasing healthcare costs. Notably, patients who were iron deficient at the time of positive FOBT results were not more likely to have a bleeding source detected on endoscopic evaluation.

Recent data have shown that 30-day readmission and rebleeding rates for patients hospitalized with overt lower GIB are $21 \%$ and $8 \%$ respectively. ${ }^{10}$ No prior studies have evaluated risk factors for either hospital readmissions or rebleeding in patients hospitalized with anemia and occult GIB. We demonstrate that the 30-day readmission rate for patients in our cohort was $28 \%$, with a majority of readmissions being unrelated to GIB. This suggests that occult GIB may a marker for chronic illness. More importantly, having either an inpatient endoscopic evaluation, or inpatient confirmation of bleeding source, was not associated with a reduced risk of rebleeding within 30 days. These results support that an inpatient endoscopic evaluation can be deferred to the outpatient setting in selected patients, given that they do not have an increased risk of recurrent bleeding.

These results must be interpreted in the context of the study design. As this was an observational study, there was no structured protocol for endoscopic evaluation or intervention. As FOBT testing is only recommended in the context of colon cancer screening and positive results require exclusion of colonic malignancy, ${ }^{2}$ we cannot exclude the presence of colonic lesions in the patients who only underwent EGD. In addition, given the relatively small numbers of patients in our study, we were likely underpowered to detect significant associations between variables and both hospital readmissions and rebleeding. Given that we identified patients at the time of gastroenterology consultation, there is also a possibility of selection bias affecting our results as we were unable to capture patients with occult bleeding who did not require gastroenterology consultation. This was a small single-center study conducted at a tertiary care academic medical center thus limiting generalizability. Finally, the yield and utilization of post hospital discharge endoscopic evaluation was not available given that our primary interests were in reporting in-hospital procedure utilization and yields.

\section{Conclusions}

In summary, for hospitalized patients with anemia and occult GIB, those with active malignancy and lower admission hemoglobin values are more likely to have an identified

Figure 1. Association of bccult bleeding score with: A) localization of bleeding source, and $B$ ) endoscopic intervention. 
source of occult bleeding on endoscopy. An occult bleeding score based on admission hemoglobin and active malignancy can predict detection of occult bleeding and need for endoscopic intervention. Inpatient endoscopic evaluation or intervention is not associated with a reduced risk of recurrent bleeding.

\section{References}

1. Rockey DC. Occult gastrointestinal bleeding. New Engl J Med 1999;341:38-46.

2. Raju GS, Gerson L, Das A, Lewis B. American Gastroenterological Association (AGA) institute technical review on obscure gastrointestinal bleeding. Gastroenterology 2007;133:1697-717.

3. Van Rijn AF, Stroobants AK, Deutekom M et al. Inappropriate use of the faecal occult blood test in a university hospital in the Netherlands. Eur J Gastroenterol Hepatol 2012;24:1266-9.

4. Sharma VK, Komanduri S, Nayyar S, et al. An audit of the utility of in-patient fecal occult blood testing. Am J Gastroenterol 2011;96:1256-60.

5. Bini EJ, Reinhold JP, Weinshel EH, et al.
Prospective evaluation of the use and outcome of admission stool guaiac testing: the Digital Rectal Examination on Admission to the Medical Service (DREAMS) study. J Clin Gastroenterol 2006;40:821-7.

6. Ip S, Sokoro AA, Kaita L, et al. Use of fecal occult blood testing in hospitalized patients: results of an audit. Can J Gastroenterol Hepatol 2014;28:489-94.

7. Rockey DC, Koch J, Cello JP, et al. Relative frequency of upper gastrointestinal and colonic lesions in patients with positive fecal occult blood tests. N Engl J Med 1998;339:153-9.

8. Liu K, Kaffes AJ. Review article: the diagnosis and investigation of obscure gastrointestinal bleeding. Alimentary Pharmacol Ther 2011;34:416-23.

9. Fry LC, Belutti M, Neumann $\mathrm{H}$ et al. Incidence of bleeding lesions within reach of conventional upper and lower endoscopes in patients undergoing double-balloon enteroscopy for obscure gastrointestinal bleeding. Aliment Pharmacol Ther 2009;29:342-9.

10. Sengupta N, Tapper EB, Patwardhan VR, et al. Risk factors for adverse outcomes after hospitalization for lower gastrointestinal bleeding. Mayo Clinic Proceedings 2015 [in press].

11. Sengupta N, Patwardhan VR, Tapper EB, et al. The risks of thromboembolism vs. recurrent gastrointestinal bleeding after interruption of systemic anticoagulation in hospitalized inpatients with gastrointestinal bleeding: a prospective study. Am J Gastroenterol 2015;110:328-35.

12. Hauser TH, Ho KK. Accuracy of on-line databases in determining vital status. $\mathbf{J}$ Clin Epidemiol Dec 2001;54:1267-70.

13. Ip S, Sokoro AA, Buchel A, et al. Use of fecal occult blood test in hospitalized patients: survey of physicians practicing in a large central Canadian health region and Canadian gastroenterologists. Can J Gastroenterol 2013;27:711-6.

14. Capurso G, Baccini F, Osborn J, et al. Can patient characteristics predict the outcome of endoscopic evaluation of iron deficiency anemia: a multiple logistic regression analysis. Gastrointestinal Endoscopy 2004;59:766-71.

15. Majid S, Salih M, Wasaya R, et al. Predictors of gastrointestinal lesions on endoscopy in iron deficiency anemia without gastrointestinal symptoms. BMC Gastroenterology 2008;8:52. 